\title{
Comparing the efficacy of metronome beeps and stepping stones to adjust gait: steps to follow!
}

\author{
Paulina J. M. Bank • Melvyn Roerdink • \\ C. E. Peper
}

Received: 25 February 2010/Accepted: 15 December 2010/Published online: 8 January 2011

(C) The Author(s) 2011. This article is published with open access at Springerlink.com

\begin{abstract}
Acoustic metronomes and visual targets have been used in rehabilitation practice to improve pathological gait. In addition, they may be instrumental in evaluating and training instantaneous gait adjustments. The aim of this study was to compare the efficacy of two cue types in inducing gait adjustments, viz. acoustic temporal cues in the form of metronome beeps and visual spatial cues in the form of projected stepping stones. Twenty healthy elderly (aged $63.2 \pm 3.6$ years) were recruited to walk on an instrumented treadmill at preferred speed and cadence, paced by either metronome beeps or projected stepping stones. Gait adaptations were induced using two manipulations: by perturbing the sequence of cues and by imposing switches from one cueing type to the other. Responses to these manipulations were quantified in terms of step-length and step-time adjustments, the percentage correction achieved over subsequent steps, and the number of steps required to restore the relation between gait and the beeps or stepping stones. The results showed that perturbations in a sequence of stepping stones were overcome faster than those in a sequence of metronome beeps. In switching trials, switching from metronome beeps to stepping stones was achieved faster than vice versa, indicating that gait was influenced more strongly by the stepping stones than the metronome beeps. Together these results revealed that, in healthy elderly, the stepping stones induced gait
\end{abstract}

Electronic supplementary material The online version of this article (doi:10.1007/s00221-010-2531-9) contains supplementary material, which is available to authorized users.

P. J. M. Bank · M. Roerdink $(\bowtie) \cdot$ C. E. Peper

Research Institute MOVE, Faculty of Human Movement

Sciences, VU University Amsterdam, Van der Boechorststraat 9,

1081 BT Amsterdam, The Netherlands

e-mail: m.roerdink@fbw.vu.nl adjustments more effectively than did the metronome beeps. Potential implications for the use of metronome beeps and stepping stones in gait rehabilitation practice are discussed.

Keywords Gait adaptability - Cueing - Stepping stones · Metronome · Treadmill walking

\section{Introduction}

Acoustic and visual cues have been used in rehabilitation practice to improve pathological gait, predominantly by means of metronome beeps and markers fixed on the floor, respectively. For metronomes, immediate and carry-over effects (i.e., from walking with to walking without cues) have been reported for walking speed, cadence, stride length, and/or symmetry of gait in patients with, for example, a cerebrovascular accident, Parkinson's disease, and traumatic brain injury (Ford et al. 2010; Hurt et al. 1998; McIntosh et al. 1997; Nieuwboer et al. 2007; Roerdink et al. 2007; Suteerawattananon et al. 2004; Thaut et al. 1997, 2007). Fixed markers on the floor, e.g., stripes or stepping stones, have been reported to increase stride length, walking velocity, and symmetry of gait in Parkinsonian patients in particular (Martin 1967; Morris et al. 1994, 1996; Lewis et al. 2000; Suteerawattananon et al. 2004).

The abovementioned studies primarily focused on cuerelated improvements in gait characteristics, whereas the potential of metronomes and spatial targets for evaluation and training of instantaneous gait adaptations has received little attention. This is unfortunate for two reasons: (1) gait adaptability, loosely defined as one's ability to modify gait (e.g., to avoid an obstacle, to change speed or direction, or 
to negotiate in cluttered terrain), is often impaired in pathological gait (e.g., Den Otter et al. 2005; Hofstad et al. 2006; Roerdink et al. 2009) and (2) restoration of gait adaptability has been associated with a lower fall risk (Weerdesteyn et al. 2006). In two recent studies, metronomes were successfully exploited to evaluate gait adaptability in stroke patients by using rhythm perturbations (Pelton et al. 2010; Roerdink et al. 2009). Specifically, those studies illustrated that external rhythms may, next to improving gait characteristics (Roerdink et al. 2007; Thaut et al. 2007), also be used to identify deficits in gait adaptability. Moreover, perturbation of the frequency or phasing of the metronome rhythm may provide a fruitful tool to train gait adaptability in rehabilitation (as discussed in Roerdink et al. 2009).

The present study was designed to compare two cueing types (an acoustic metronome and visual spatial targets) with respect to their relative efficacy in triggering instantaneous gait adjustments. So far, no firm statement can be made in this regard (Lim et al. 2005; Rubinstein et al. 2002), mainly due to methodological limitations related to overground walking. First, gait examination during overground walking is typically limited to a small number of strides, capturing just a fragment of gait attuned to the particular type of cues. Hence, the coordination between gait and those cues cannot be assessed reliably. Second, metronomes and spatial targets typically affect different aspects of gait during overground walking: metronomes explicitly prescribe cadence, whereas spatial targets explicitly prescribe step length. Although these explicit prescriptions may implicitly affect other gait parameters as well (e.g., constraining cadence may also affect step length and walking velocity), variations in these resulting gait parameter relations (Bertram and Ruina 2001) render direct comparisons problematic. Despite recent attempts (e.g., Amatachaya et al. 2009; Suteerawattananon et al. 2004), these limitations have hampered the examination of the relative effects of available cueing types on pathological gait.

To overcome these methodological limitations, our comparisons were based on treadmill walking. Using a treadmill, a relatively large number of steps could be examined in relation to metronome beeps or stepping stones. At the same time, the discrepancy between the two types of cues in terms of the prescribed gait parameters was circumvented by imposing a fixed walking speed. Because walking velocity $(v)$, step frequency $(f)$, and step length $(l)$ are related according to $v=f \times l$, prescription of the step frequency (by means of an acoustic metronome) implicitly prescribes step length as well if a fixed walking speed is imposed. Conversely, if walking speed is fixed, prescription of step length (by means of projected stepping stones) also determines step frequency. Hence, we were able to invoke the same requirements with regard to gait parameters (viz. walking velocity, step frequency, and step length) for either type of cues.

We examined a group of healthy elderly in the same age range as most patients involved in gait rehabilitation training. The relative efficacy of metronome beeps and stepping stones in inducing instantaneous gait adjustments was assessed. To this end, we examined two types of manipulations: perturbations of the sequence of cues and switches from one cueing type to the other. In perturbation trials, the sequence of metronome beeps or stepping stones was perturbed by suddenly changing a particular interval between subsequent cues. The time needed to overcome those perturbations indicates the efficacy of the specific cues to elicit the required gait adaptation. In switching trials, participants were instructed to switch voluntarily from coordination with metronome beeps to coordination with stepping stones, or vice versa. These switches required a gait adjustment, because the two cueing sequences were phase-shifted with respect to one another. The time needed to perform the switch provides a relative index of the degree to which gait is influenced by the specific cueing type (Kelso et al. 1988), allowing for comparison of the ease with which the steps were tuned to either metronome beeps or stepping stones.

To restore coordination with the cues after either type of manipulation, participants had to adapt gait by briefly accelerating or decelerating their steps, requiring instantaneous changes in both step length and step time. Because visual information has been argued to be the most important and ecologically salient source of information for walking (under normal circumstances, for individuals without visual impairments; Hollands et al. 2002; Kennedy et al. 2003; Patla 1997), we hypothesized that stepping stones would induce such self-initiated gait adjustments more effectively than metronome beeps. Previous research indicated that voluntary acceleration and deceleration of steps differed with regard to their dependence on specific neural mechanisms (associated with corticospinal pathways vs. supraspinal structures; Varraine et al. 2000). Therefore, perturbation and switching manipulations were configured in such a way that both longer-step and shorter-step responses (i.e., requiring instantaneous lengthening or shortening, respectively, of both step time and step length) were induced by either type of manipulation (see Methods below). This general method to delineate relative difficulties or deficits in particular step adjustment strategies has been pursued successfully in recent experiments on rhythm perturbations (Pelton et al. 2010; Roerdink et al. 2009) and obstacle avoidance (e.g., Den Otter et al. 2005; Hofstad et al. 2006; Weerdesteyn et al. 2005a, b). Based on the results of these behavioral studies, we expected that also in our experiment participants would show more difficulty in 
gait adaptations requiring a shorter-step response (i.e., requiring a shortening of both step time and step length).

\section{Methods}

Subjects

After providing informed consent, twenty healthy elderly (10 women; age: $63.2 \pm 3.6$ years) participated in the study, which was approved by the local ethics committee. Participants were excluded if they reported cardiovascular or cardiopulmonary problems, orthopedic conditions, use of walking aids, any other condition that limits mobility, and/or uncorrected visual or auditory impairment.

\section{Apparatus}

A treadmill with a large force platform embedded (ForceLink, Culemborg, the Netherlands) was used, allowing for online detection of gait events (e.g., heel strike, midstance) and gait characteristics (e.g., cadence, step length) (Roerdink et al. 2008). The instrumented treadmill was equipped with custom-made software for presentation of metronome beeps and stepping stones contingent upon online gait registrations (Roerdink 2008). During all trials, a long projection board $(1.80 \mathrm{~m})$ with the same appearance as the treadmill surface was attached to the front of the treadmill (Fig. 1). In the stepping stones conditions, 4-5 stepping stones (dimensions: shoe length by $30 \mathrm{~cm}$ ) were projected on the projection board and treadmill, such that the upcoming stepping stones were visible to the participant (Fig. 1). The speed at which the stepping stones approached the participant corresponded to the belt speed. The stepping stones were presented to the left and right to cue left and right footfalls, respectively. In the metronome conditions, computer-generated rhythmic acoustic stimuli were administered through earphones to cue the left $(440 \mathrm{~Hz})$ and right $(1,000 \mathrm{~Hz})$ footfalls.

\section{Procedure}

\section{Familiarization}

Participants first practiced walking on the treadmill for at least $10 \mathrm{~min}$ to warrant similar gait characteristics to overground walking (Parvataneni et al. 2009; Wass et al. 2005). For each participant, the most comfortable treadmill walking speed (CTWS) was determined $(4.5 \pm 0.3 \mathrm{~km} / \mathrm{h})$. Next, participants practiced walking with either cueing type (metronome or stepping stones; order counterbalanced over participants). Cues were presented at seven frequencies (ranging from 77.5 to $122.5 \%$ of the preferred cadence

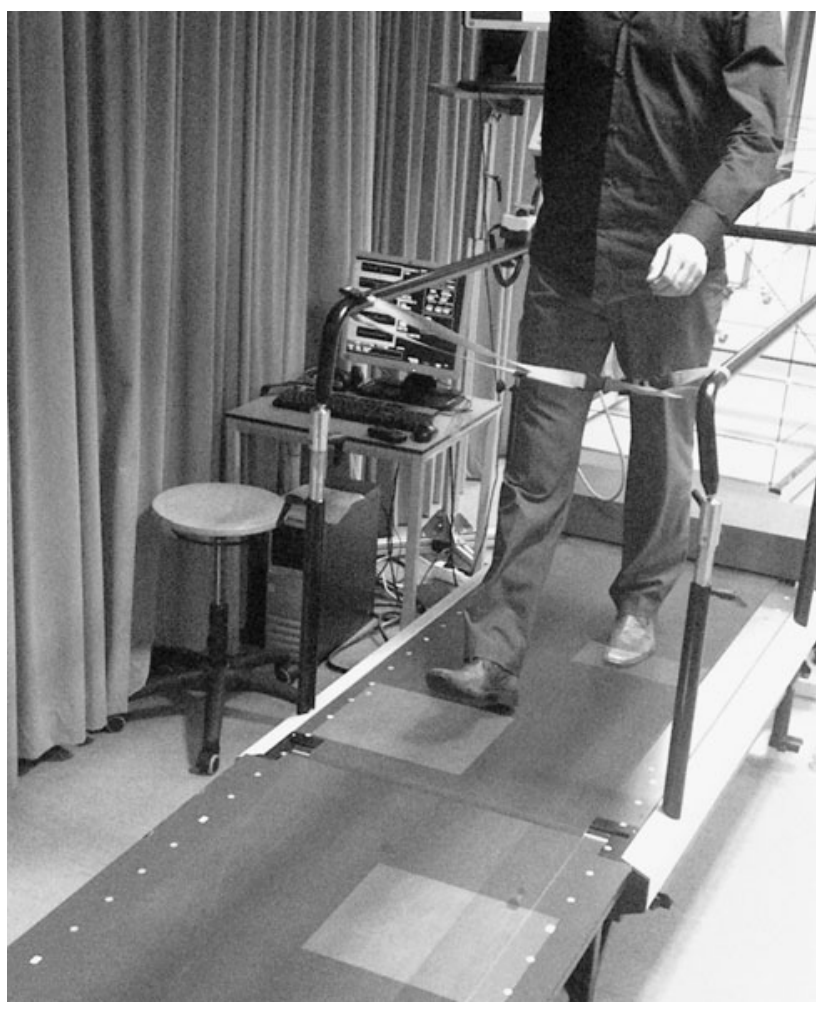

Fig. 1 Overview of the experimental setup (i.e., stepping stones condition)

at CTWS, in steps of 7.5\%; presented in random order) for 36 strides each, separated by approximately $15 \mathrm{~s}$ of uncued walking.

\section{Perturbation trials}

Belt speed was set at the participant's CTWS. For each individual trial, the preferred cadence was determined online based on the last three strides (i.e., six steps) of uncued walking. Next, metronome beeps or stepping stones corresponding to the participant's preferred cadence/step length were presented for about $25 \mathrm{~s}$. At a random time instant after 8-12 s of cued walking, a perturbation was applied to the sequence of cues. Participants were instructed to neutralize the effects of the perturbation as fast as possible by adjusting gait.

For the metronome condition, the perturbation entailed a sudden temporal shift of $\pm 1 / 6$ th of the interval between consecutive ipsilateral beeps $\left(T_{\text {cue }}\right)$, resulting in a $+60^{\circ}$ (phase delay) or $-60^{\circ}$ (phase advance) shift of all subsequent beeps. Gait adjustment was required to restore synchronization with the metronome: typically a longerstep response (a temporarily larger step time and step length) for a $+60^{\circ}$ phase shift and a shorter-step response (a temporarily smaller step time and step length) for a $-60^{\circ}$ phase shift (Roerdink et al. 2009). For the stepping 
stones condition (in which multiple upcoming stepping stones were visible), phase-delay perturbations were administered by temporarily arresting the stepping stones at the predicted time instant of heel strike $\left(t_{H S \text {,predicted }}\right)$ and resuming their movement after $T_{\text {cue }} / 6$ (equivalent to a displacement of $1 / 6$ th of the stride length). The $-60^{\circ}$ phase shift was realized by suddenly displacing the projected stepping stones in the direction toward the participant over a distance of $1 / 6$ th of the stride length at $T_{\text {cue }} / 6$ before $t_{H S \text {,predicted }}$ Thus, perturbations in metronome beeps and stepping stones conditions were compatible in terms of timing, size, and unpredictability. Figure $2 \mathrm{a}$, d provide a schematic of these perturbations (videos of representative trials of these different conditions are available in Online Resource 1).

Phase-delay and phase-advance perturbations were delivered to the left or right foot. The trials were grouped in 'cueing type' blocks (order counterbalanced over participants). Four trials were conducted for each condition, yielding 16 perturbation trials per cueing type block ( 2 [left vs. right foot] $\times 2$ [phase advance vs. phase delay] $\times 4$ repetitions). In addition, each block comprised three dummy trials, in which no perturbation was administered. Within the cueing type blocks, trials were presented in random order.

\section{Switching trials}

Belt speed was set at the participant's CTWS. For each trial, the preferred cadence was determined online based on the last three strides of uncued walking. The participant continued walking, now paced by either metronome beeps or stepping stones at this preferred cadence. At a random time instant after 8-12 s of cued walking, cues of the other

a
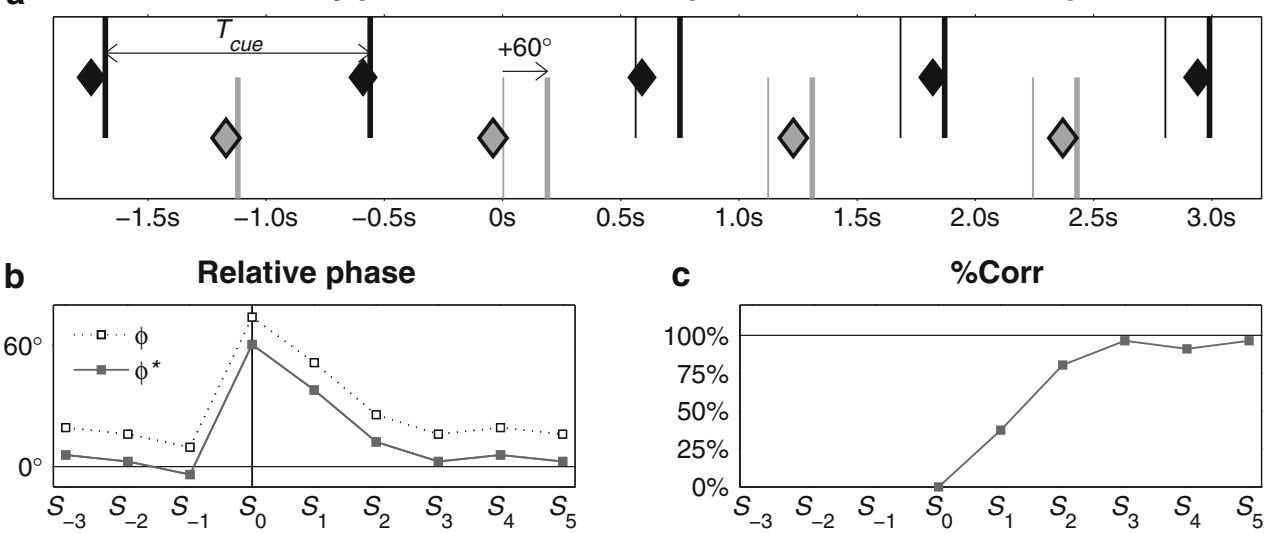

C

$\%$ Corr

d

Phase delay perturbation in the sequence of stepping stones

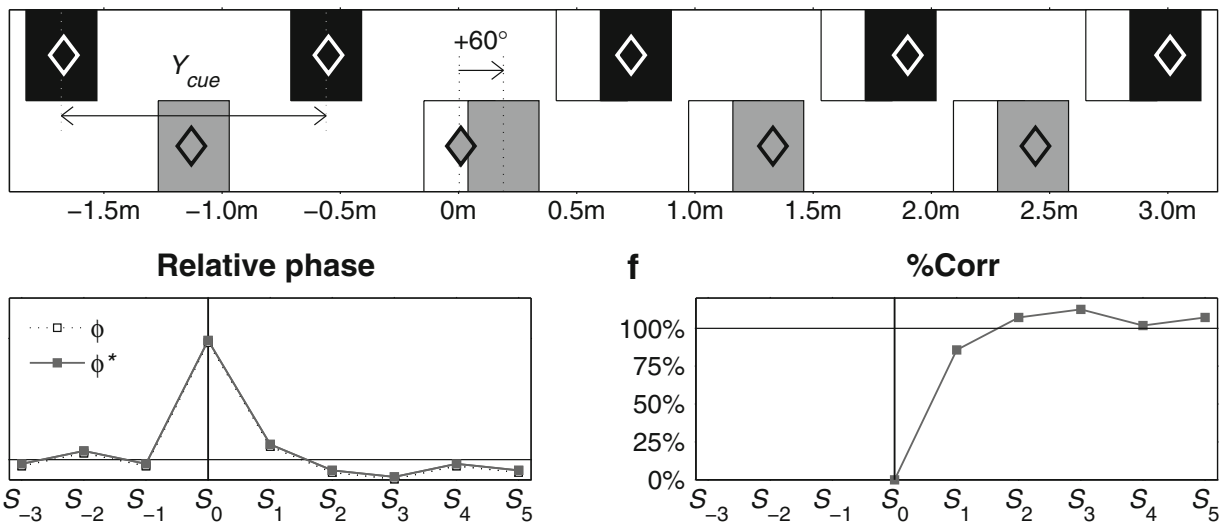

Fig. 2 Schematic representation of phase-delay $\left(+60^{\circ}\right.$ phase shifts) perturbations in the sequence of metronome beeps (a gray and black thick lines indicate metronome onsets and thin line segments represent beeps if the perturbation would not have occurred) and stepping stones (d gray and black patches indicate projected stepping stones and the partly occluded white patches represent stepping stones if the perturbation would not have occurred). For both cueing conditions, representative gait events are indicated by diamond markers (representing time instants of heel strike and the center position of the foot in a and $\mathbf{d}$, respectively). In this schematic representation, belt speed was $1.0 \mathrm{~m} / \mathrm{s}$, so $m$ in (d) is comparable to $s$ in (a). b and e depict corresponding relative phase time series $\phi$ and $\phi^{*}$ for $S_{-3}$ to $S_{5}$ for metronome and stepping stones conditions, respectively; note that in (e) $\phi \approx \phi^{*}$. $\mathbf{c}$ and $\mathbf{f}$ depict associated \%Corr for $S_{0}-S_{5}$ for metronome and stepping stones conditions, respectively. See text for further details 
type were added for another $20 \mathrm{~s}$ with a phase shift of $\pm 90^{\circ}$ with respect to the initial cues. Participants were instructed to match their steps with the new cues as fast as possible, which typically required a gait adjustment of $-90^{\circ}$ (shorter-step response) or $+90^{\circ}$ (longer-step response). Two switching directions were examined: from stepping stones to metronome beeps (StoM) and vice versa (MtoS). To avoid that the stepping stones would be ignored after the switch in StoM conditions (which would result in an unfair comparison with condition MtoS, in which the metronome beeps could not be ignored), participants were instructed to keep on looking at the belt throughout all trials (i.e., irrespective of switching direction). Participants lightly touched the handrails at a marked position to prevent positional changes on the treadmill, which would affect the phasing between the two types of cues. The StoM and MtoS switching directions are visualized in Online Resource 2.

Eight trials were conducted for each switching direction (StoM and MtoS), four for each phase shift $\left(-90^{\circ}\right.$ and $+90^{\circ}$ ). These 16 trials were presented in four blocks, containing one trial of each condition presented in random order. These blocks were conducted after the perturbation trials had been completed.

\section{Data analysis}

For each trial, the relative phase $\phi\left(\right.$ in $^{\circ}$ ) between gait and the cues was calculated for each step on the basis of online registered gait event data. The gait events in question (viz., heel strikes and midstances) were derived from center-ofpressure (COP) characteristics (for more details, see Roerdink et al. 2008). The fixed relationship between belt speed, cadence, and step length enabled us to determine $\phi$ in a compatible manner for both cueing conditions (cf. Fig. 2b, e). Specifically, for the metronome conditions, $\phi$ was defined as $\phi=360^{\circ} \cdot\left(t_{\text {cue }}-t_{H S}\right) / T_{\text {cue }}$, with $t_{\text {cue }}$ (in ms) denoting the time instant of beep onset, $t_{H S}$ denoting the time instant of the corresponding heel strike, and $T_{\text {cue }}$ denoting the time interval between consecutive ipsilateral beeps (Roerdink et al. 2007). For the stepping stones conditions, $\phi$ was determined in a similar manner by relating foot position (fore-aft COP position at midstance) to the center position of the stepping stone. Thus, after replacing time instants $t$ by positions (on the treadmill) $y$ (in $\mathrm{mm}$ ), $\phi=360^{\circ} \cdot\left(y_{\text {cue }}-y_{\text {foot }}\right) / Y_{\text {cue }}$, with $y_{\text {cue }}$ and $y_{\text {foot }}$ denoting the fore-aft coordinates of the centers of the stepping stone and the corresponding foot, respectively, and $Y_{\text {cue }}$ the distance between consecutive ipsilateral stepping stones.

For each trial, pre-manipulation (i.e., pre-perturbation or pre-switch) coordination between gait and cues was quantified, using the five strides immediately preceding the perturbation or switch. Specifically, we quantified premanipulation coordination in terms of the mean relative phase $\left(\bar{\phi}_{\text {pre }}\right)$, its standard deviation $\left(\sigma_{\phi, \text { pre }}\right)$, and the cumulative phase error ( $P E$; defined as the sum of successive increments/decrements in $\phi$ : large values of $|P E|$ indicate the presence of systematic drift or 'phase wrapping'). Post-manipulation performance was quantified by means of $\bar{\phi}_{\text {post }}$ and $\sigma_{\phi \text {,post }}$, as obtained for the postmanipulation window of five strides for which $|P E|$ was minimal. Trials were excluded from analysis if (a) gait was not sufficiently matched to the pacing frequency prior to the manipulation $\left(\left|P E_{\text {pre }}\right|>54^{\circ}\right.$, corresponding to a drift $>115 \%$ of the stride cycle); (b) the initial response was in the wrong direction $\left(|\phi|>\left(|\Delta \phi|+45^{\circ}\right)\right.$ for more than one step, with $\Delta \phi$ representing the phase shift, i.e., $\pm 60^{\circ}$ or $\pm 90^{\circ}$ for perturbation or switching trials, respectively); (c) the post-manipulation relative phase deviated considerably from the required relative phase (for perturbation trials: $\left|\bar{\phi}_{\text {post }}-\bar{\phi}_{\text {pre }}\right|>45^{\circ}$, implying unsuccessful return to the pre-perturbation phase relation; for switching trials: $\left|\bar{\phi}_{\text {post }}\right|>45^{\circ}$, implying unsuccessful adjustment to the new cues $\left(\bar{\phi}_{\text {post }}\right.$ here represents the phase relation with the new cues)); or (d) no stable post-manipulation coordination was achieved (there was no post-manipulation episode of at least 6 consecutive steps during which $\bar{\phi}$ fell within its reference range; see running window analysis below). Based on these criteria, 40 perturbation $(6 \%)$ and 80 switching $(25 \%)$ trials were excluded. The distribution of these trials varied markedly over the cueing type conditions (Table 1).

A running window analysis was performed to determine the number of steps required to achieve stable post-manipulation synchronization to the cues $\left(N_{\text {return }}\right.$ for perturbation trials and $N_{\text {switch }}$ for switching trials). For each post-manipulation window of three steps, mean relative phase $\bar{\phi}$ was calculated. For perturbation trials, $N_{\text {return }}$ corresponded to the first window for which $\bar{\phi}$ fell within the reference range $\bar{\phi}_{\text {post }} \pm 1.96 \cdot \sigma_{\phi \text {,pre }}$ and stayed within this range for at least

Table 1 Distribution of excluded trials over conditions

\begin{tabular}{lllllll}
\hline Perturbation condition & & & \multicolumn{2}{l}{ Switching condition } \\
\cline { 1 - 2 } \cline { 6 - 7 } Conditions & $\begin{array}{l}\text { Number } \\
\text { of trials }\end{array}$ & $\chi^{2}(1)$ & & Conditions & $\begin{array}{l}\text { Number } \\
\text { of trials }\end{array}$ & $\chi^{2}(1)$ \\
\hline $\mathrm{M} / \mathrm{S}$ & $36 / 4$ & $8.5^{* *}$ & & StoM/MtoS & $73 / 7$ & $45.4^{* *}$ \\
$+60^{\circ} /-60^{\circ}$ & $21 / 19$ & 0.03 & & $+90^{\circ} /-90^{\circ}$ & $28 / 52$ & $6.0^{*}$ \\
\hline
\end{tabular}

Significant $\chi^{2}$-values indicate that excluded trials are unevenly distributed over conditions

$M$ refers to the metronome condition, while $S$ refers to the stepping stones condition

$* P<.05, * * P<.01$ 
six consecutive windows. $N_{\text {return }}$ was equated with the middle step of this first window. A similar analysis was performed to determine $N_{\text {switch }}$ for switching trials, albeit that the reference range for the new cueing type was based on mean $\sigma_{\phi \text {,pre }}$ as obtained for the pre-switch parts of the eight trials in the reverse switching condition.

The time course of gait adjustments to restore coordination after perturbations or to match steps with the new, phase-shifted cues in switching trials was further quantified by means of the percentage correction of the phase error (\%Corr) for the 20 steps following the manipulation (Chen et al. 2006; Pelton et al. 2010; Roerdink et al. 2009). First, $\phi$ was determined for steps $S_{-10}$ to $S_{20}$, in which $S_{0}$ represents the step corresponding to the onset of the manipulation and $S_{ \pm n}$ represents the $n$th step preceding or following $S_{0}$. Subsequently, $\phi$ was normalized (yielding $\phi^{*}$ ) by subtracting baseline $\bar{\phi}$ (obtained for $S_{-10}$ to $S_{-1}$ ), so that $\Delta \phi$ at $S_{0}$ was $\pm 60^{\circ}$ (perturbation trials) or $\pm 90^{\circ}$ (switching trials). This was necessary in view of the phase lead typically observed for acoustically paced walking (McIntosh et al. 1997; Pelton et al. 2010; Roerdink et al. 2009). Then, for each $n$th step after the perturbation, \%Corr was defined as: $\% \operatorname{Corr}(n)=100 \% \cdot\left(\phi^{*}\left(S_{0}\right)-\phi^{*}\left(S_{n}\right)\right) / \Delta \phi$, with $\Delta \phi$ $= \pm 60^{\circ}$ (see also Fig. 2c, f). Likewise, for the switching trials, \%Corr relative to the new cues $\left(\% \mathrm{Corr}_{\text {new }}\right)$ was calculated for $S_{1}-S_{20}$, with $\Delta \phi= \pm 90^{\circ}$.

Supplementary to the analyses in terms of relative phase, for the perturbation trials also temporal (step time) and spatial (step length) gait parameters were quantified to establish how gait was adjusted in the 20 post-perturbation steps $\left(S_{1}-S_{20}\right)$ to restore coordination with the cues. To facilitate comparison between trials and participants, step times and step lengths were normalized to the step time and step length as prescribed by the sequence of cues. Specifically, normalized step time was defined as $T_{\text {step }}=100 \% \cdot\left(t_{H S, n+1}-t_{H S, n}\right) /\left(0.5^{*} T_{\text {cue }}\right)$, while normalized step length was defined as $Y_{\text {step }}=100 \% \cdot\left(y_{\text {foot }, n+1}-y_{\text {foot }, n}\right) /\left(0.5^{*} Y_{\text {cue }}\right)$, in which $t_{H S, 0}$ and $y_{\text {foot }, 0}$ denote the time instant of the heel strike and the fore-aft position of the foot corresponding to the onset of the perturbation, respectively, and $n$ denotes step number (ranging from 0 to 20).

\section{Statistical analysis}

In view of empty cells resulting from the exclusion criteria, perturbation data of one participant and switching data of six participants were excluded from statistical analysis. For the remaining participants, $T_{\text {step }}, Y_{\text {step }}, \%$ Corr, and $\%$ Corr $_{\text {new }}$ were averaged per condition, for each $S_{n}$. The time course of the response was examined by subjecting $T_{\text {step }}, Y_{\text {step }}$, \%Corr, and $\%$ Corr $_{\text {new }}$ to a 20 (post-manipulation step number; $\left.S_{n}\right) \times 2$ (cueing type: metronome vs. stepping stones; or switching direction: StoM vs. MtoS) $\times 2$ (phase shift: advance vs. delay) repeated-measures analysis of variance (ANOVA). Because this analysis was conducted to examine the time course of gait adaptation, only effects that involved the factor step-number were reported. For the time-independent measures $N_{\text {return }}$ and $N_{\text {switch }}$, the median values (to reduce the influence of outliers) per condition were subjected to a 2 (cueing type or switching direction) $\times 2$ (phase shift) repeated-measures ANOVA. Degrees of freedom were adjusted in case the sphericity assumption was violated (Field 2005). Effect size was quantified as $\eta_{p}^{2}$. For significant interaction effects $(P<.05)$, post hoc analyses were performed using two-tailed paired-samples $t$ tests, using the modified Bonferroni test (Keppel 1991). Values are presented as mean \pm standard deviation.

\section{Results}

\section{Perturbation}

For both $T_{\text {step }}$ and $Y_{\text {step }}$, a significant main effect of step number was observed $(F(3.48,62.64)=5.02, \quad P<.05$, $\eta_{p}^{2}=.22$ and $F(4.23,76.21)=3.41, P<.05, \quad \eta_{p}^{2}=.16$, respectively), as well as interactions between phase shift and step number $\left(F(2.58,46.52)=174.65, P<.001, \eta_{p}^{2}=.91\right.$ and $F(3.14,56.44)=141.75, P<.001, \eta_{p}^{2}=.89$, respectively), cueing type and step number $(F(4.29,77.26)=5.34$, $P<.001, \eta_{p}^{2}=.23$ and $F(4.74,85.24)=5.28, P<.001$, $\eta_{p}^{2}=.23$, respectively), and cueing type, phase shift, and step number $\left(F(3.68,66.25)=29.15, P<.001, \eta_{p}^{2}=.62\right.$ and $F(4.75,85.54)=58.14, P<.001, \eta_{p}^{2}=.76$, respectively). Post hoc analysis of the three-way interactions revealed that phase-delay and phase-advance perturbations induced different step adjustment responses. As can be appreciated from Fig. 3 , phase-advance perturbations $\left(-60^{\circ}\right.$ phase shift $)$ induced a decrease in step time and step length (i.e., a shorterstep response; $T_{\text {step }}$ and $Y_{\text {step }}<100 \%$ ), whereas phase-delay perturbations $\left(+60^{\circ}\right.$ phase shift $)$ induced an increase in step time and step length (i.e., a longer-step response; $T_{\text {step }}$ and $\left.Y_{\text {step }}>100 \%\right)$. For the stepping stones condition, the difference between the responses to the phase-advance and phasedelay perturbations was significant for $S_{1-4}$ and $S_{1-3}$ for $T_{\text {step }}$ and $Y_{\text {step }}$, respectively, whereas for the metronome condition it was significant for $S_{1-5}$ and $S_{1-6}$ for $T_{\text {step }}$ and $Y_{\text {step }}$, respectively. ${ }^{1}$ Moreover, the time course and magnitude of these step

\footnotetext{
${ }^{1}$ In addition, some significant differences were obtained at higher step numbers for both $T_{\text {step }}$ and $Y_{\text {step. }}$. However, these differences varied in sign and were all smaller than $2.5 \%$ of the prescribed step time and length, respectively. Therefore, these additional post hoc results were considered immaterial for the interpretation of the threeway interaction.
} 
Fig. 3 Mean normalized step times (a, $\left.T_{\text {step }}\right)$ and step lengths (b, $Y_{\text {step }}$ ) for $S_{1}-S_{20}$ for the perturbation conditions. Note that following phase-advance $\left(-60^{\circ}\right)$ perturbations, a shorterstep response was observed (with both $T_{\text {step }}$ and $Y_{\text {step }}<100 \%$ regardless of the cueing type); after phase-delay $\left(+60^{\circ}\right)$ perturbations, a longerstep response was observed (with both $T_{\text {step }}$ and $Y_{\text {step }}>100 \%$ regardless of the cueing type). For both phaseshift directions, significant differences between cueing types for a given $S_{\mathrm{n}}$ are indicated by an open symbol for the $T_{\text {step }}$ or $Y_{\text {step }}$ that deviated most from $100 \%$; initial steptime and step-length adjustments were faster and larger for the stepping stone $(S)$ than metronome beep $(M)$ conditions

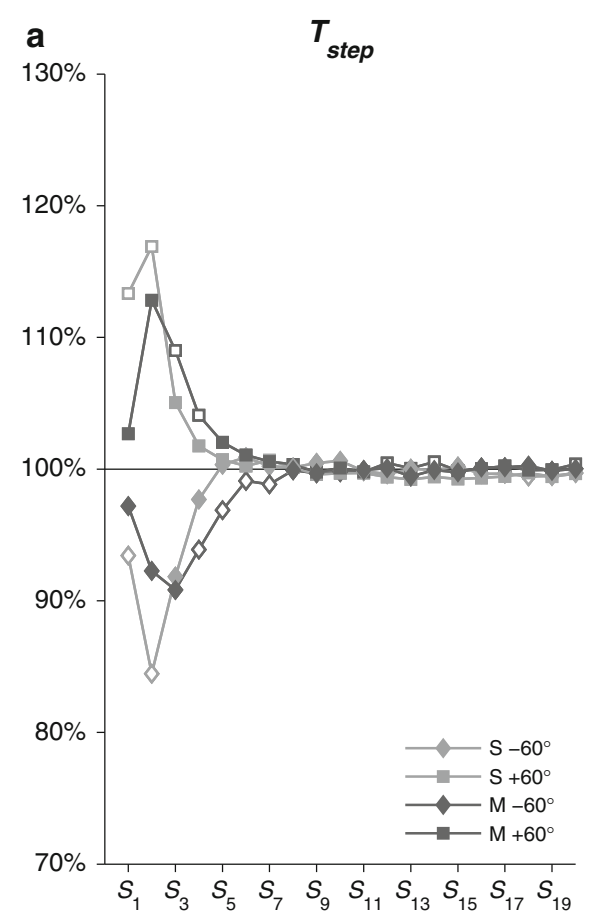

adjustments differed between metronome beeps and stepping stones conditions (Fig. 3, open symbols). Specifically, in the stepping stones conditions, the initial step-time and steplength adjustments were larger and occurred faster than in the metronome conditions, where step adjustments took place in a more gradual fashion.

Figure $4 \mathrm{a}, \mathrm{b}$ presents individual and mean relative phase responses to the applied perturbation manipulations for all participants included in the statistical analysis. As can be appreciated from these figures, the stepping stones were more effective in inducing step adjustments than were the metronome beeps. Indeed, $N_{\text {return }}$ was significantly smaller for the stepping stones condition $(2.9 \pm 1.1$ steps $)$ than for the metronome condition $(6.4 \pm 1.5$ steps $), F(1,18)=$ 87.45, $P<.001, \eta_{p}^{2}=.83$ (cf. Fig. 4a, b).

For \%Corr, a significant main effect of step number, $F(19,342)=218.09, P<.001, \eta_{p}^{2}=.78$, and interactions between cueing type and step number, $F(19,342)=$ 122.30, $P<.001, \eta_{p}^{2}=.87$, and cueing type, phase shift, and step number, $F(19,342)=3.76, P<.001, \eta_{p}^{2}=.17$, were obtained. Post hoc analysis of the three-way interaction revealed significant changes in \%Corr with step number for all conditions, reflecting successful gait adaptation over a number of steps (Fig. 5a). For both perturbation directions, \%Corr was significantly larger in the stepping stones condition than in the metronome condition in the first 13 steps, except for $S_{10}$ and $S_{12}$ after $-60^{\circ}$ and $+60^{\circ}$ phase shifts, respectively (Fig. 5a). Only in the stepping stones condition, the direction of the phase shift significantly affected \%Corr, with \%Corr at $S_{1}$ and $S_{10}$ being larger for phase-delay than phase-advance perturbations (Fig. 5a, open symbols).

\section{Switching}

$N_{\text {switch }}$ was significantly smaller for MtoS $(6.7 \pm 2.9$ steps $)$ than for StoM switches $(9.3 \pm 3.1$ steps $), F(1,13)=5.20$, $P<.05, \eta_{p}^{2}=.29$ (cf. Fig. 4c, d). For \% Corr $_{\text {new }}$, the effect of step number, $F(2.5,32.6)=116.02, P<.001, \eta_{p}^{2}=.90$, and the interactions between switching direction and step number, $F(2.7,34.6)=16.07, P<.001, \eta_{p}^{2}=.55$, phase shift and step number, $F(2.6,33.7)=4.19, P<.05, \eta_{p}^{2}=.24$, and switching direction, phase shift, and step number, $F(2.5,31.9)=$ 7.61, $P<.001, \eta_{p}^{2}=.37$, were significant.

Post hoc analysis of the three-way interaction revealed an evolution toward coordination with the new, phaseshifted cues with step number for all conditions (Fig. 5b). In the first five steps following the introduction of the new cueing type, \% Corr $_{\text {new }}$ was larger for MtoS than for StoM conditions, irrespective of the sign of the phase shift (Fig. 5b). However, for both conditions, the subsequent time course of the switch was affected by the direction of the phase shift: in StoM conditions, \% Corr $_{\text {new }}$ was larger after $+90^{\circ}$ than after $-90^{\circ}$ phase shifts $\left(S_{3}\right)$, whereas the opposite was observed for MtoS switches $\left(S_{3}, S_{4}\right.$; Fig. 5 b, open symbols). Whereas the value of $\% \mathrm{Corr}_{\text {new }}$ around which steady post-switch coordination was realized $\left(S_{10^{-}}\right.$ $S_{20}$ ) was independent of the direction of the phase shift in the MtoS conditions, it was affected by the direction of the 
Fig. 4 Relative phase time series for $S_{-10}$ to $S_{20}$ for perturbation $(\mathbf{a}, \mathbf{b})$ and switching (c, d) conditions. Thin and thick lines represent individual and averaged relative phase time series, respectively. Dark and bright thick gray lines represent relative phase time series of footfalls with metronome beeps $(M)$ and stepping stones $(S)$, respectively

Fig. 5 Mean percentage correction for $S_{1}-S_{20}$ for perturbation (a) and switching (b) conditions. Open symbols represent significant differences between phase shift directions (advance vs. delay) a

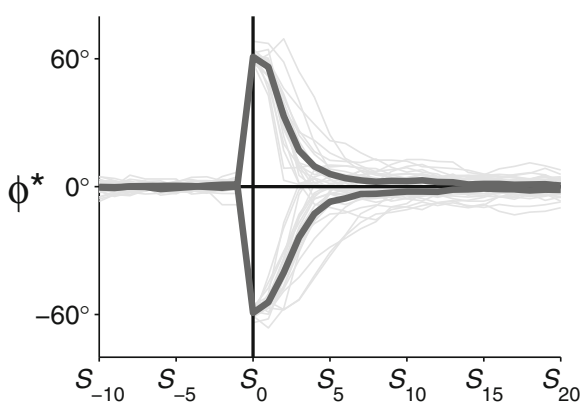

C

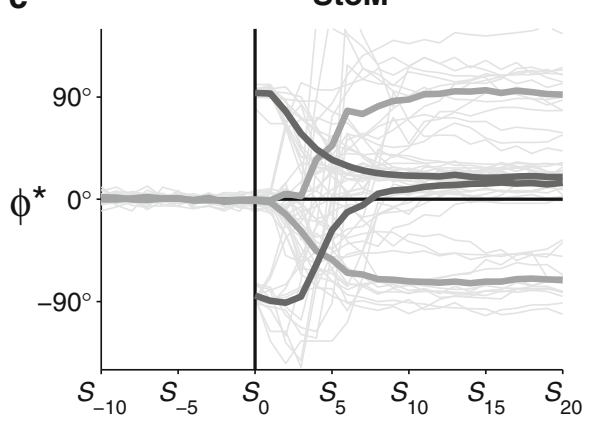

StoM

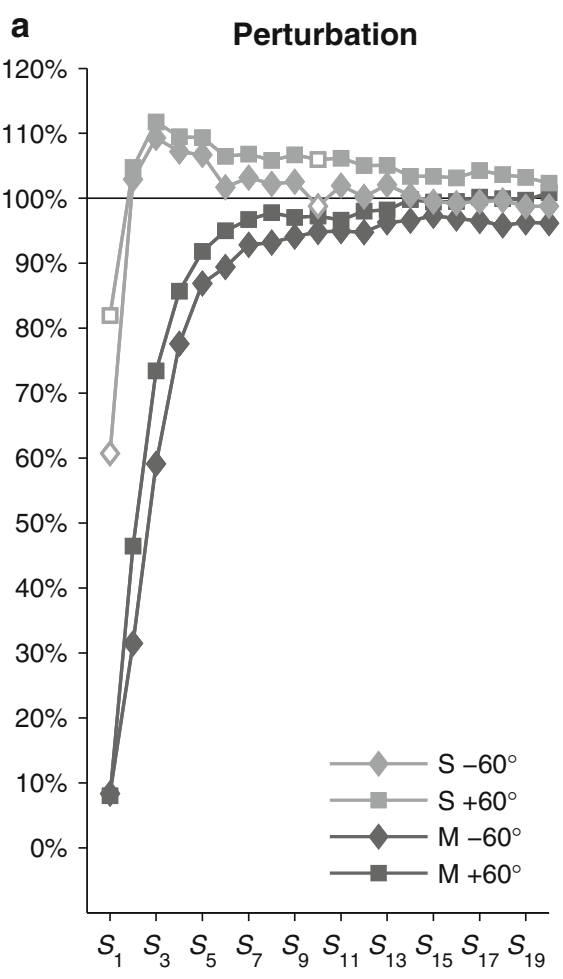

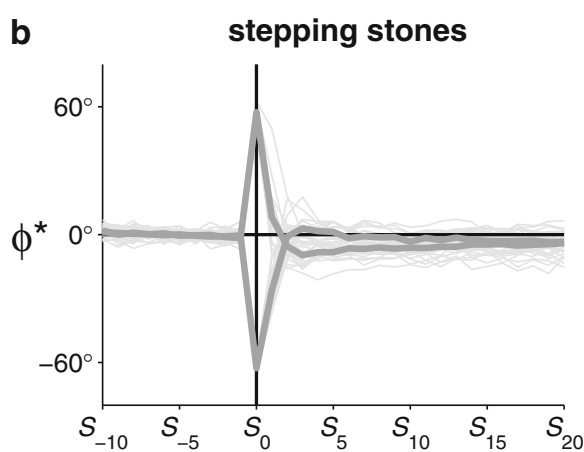

d

Mtos

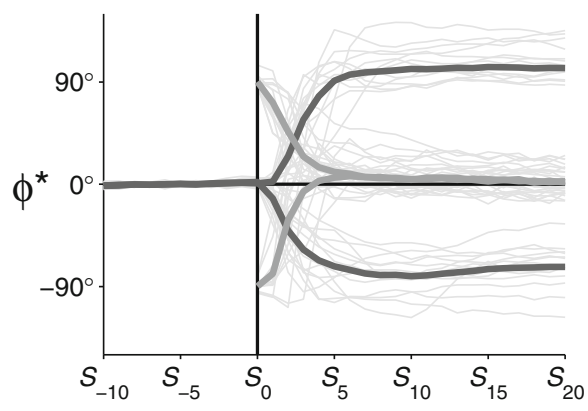

b

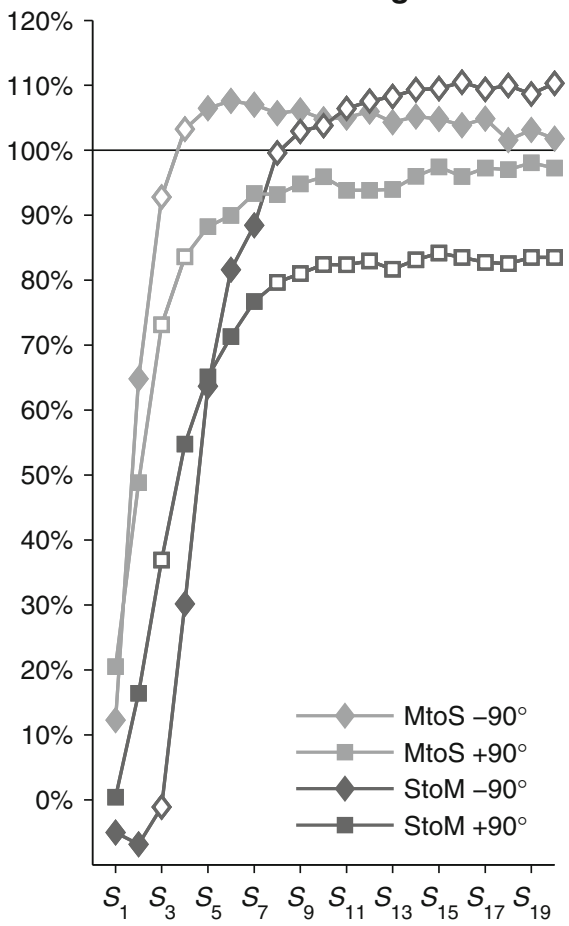

phase shift in the StoM conditions: after $+90^{\circ}$ and $-90^{\circ}$ phase shifts $\%$ Corr $_{\text {new }}$ was $83.0 \pm 13.7 \%$ and $109.0 \pm$ $8.6 \%$, respectively, which corresponded to average gait adjustments of $75^{\circ}$ and $98^{\circ}$ (Fig. 5b). These under- and overcompensations for StoM switches simply reflected a consistent tendency to time the steps in advance of the metronome beeps (cf. $\phi^{*}>0$ in Fig. 4c; see also McIntosh et al. 1997; Pelton et al. 2010; Roerdink et al. 2007, 2009). 


\section{Discussion}

This study assessed the relative efficacy of metronome beeps and stepping stones in inducing gait adaptations in healthy elderly. Since in particular visual information has been argued to comprise important and salient information for the control of walking (Hollands et al. 2002; Kennedy et al. 2003; Patla 1997), we expected that stepping stones would be more effective in triggering gait adjustments than metronome beeps. This was indeed the case. First, the results of the perturbation trials revealed that coordination with the cues was restored more rapidly in the stepping stones conditions than in the metronome conditions, as indicated by a smaller $N_{\text {return }}$ and a larger \%Corr over the first steps ${ }^{2}$ following the perturbation (Figs. $4 \mathrm{a}, \mathrm{b}$, and $5 \mathrm{a}$ ). The associated analyses of step-time and step-length adjustments showed that after phase-delay (phase-advance) perturbations both step lengths and step times were elongated (shortened) over subsequent steps, irrespective of the type of cues. However, adjustments in step length and step time were larger and occurred faster following perturbations in a sequence of stepping stones than following perturbations in a sequence of metronome beeps (Fig. 3a, b). Second, the results of the switching trials revealed that adequate adaptation to the new, phase-shifted cues was achieved faster when switching from metronome beeps to stepping stones (MtoS) than vice versa (StoM), as evidenced by a smaller $N_{\text {switch }}$ and a larger $\%$ Corr ${ }_{\text {new }}$ over the first steps following the introduction of the stepping stones (Figs. 4c, d, and 5b). Third, the distribution of excluded trials (Table 1) indicated that participants had less difficulty with post-manipulation adaptation to stepping stones than to metronome beeps. Together, these converging findings supported the hypothesis that, in healthy elderly, gait adjustments were induced more effectively by the stepping stones than by the metronome beeps.

The fact that spatial positions were presented for the required foot placement positions may be the key to understanding why gait adaptations were more effective in response to the visual stepping stones. Hollands and colleagues (Chapman and Hollands 2010; Hollands et al. 1995; Hollands and Marple-Horvat 2001) demonstrated a tight link between gaze and foot placement in young and elderly adults walking on stepping stone targets. Their results suggested that vision was predominantly used in a feedforward manner to guide and regulate stepping movements. Since the stepping stones functioned as visual

\footnotetext{
2 In this context, in particular the differences in \%Corr between the cueing conditions over the first 6 steps are noteworthy, because the differences obtained for $S_{7}-S_{13}$ seemed to be due to an overcorrection in the visual cueing condition, which was gradually absorbed in subsequent steps.
}

targets, they may thus have provided a strong reference for controlling, and thereby influencing, gait.

Furthermore, our results testified to the potential of perturbations in a sequence of cues for evaluating and training gait adjustments, most compellingly so for the stepping stones. \%Corr was found to be larger following phase-delay than phase-advance shifts of the stepping stones, in particular for the first step after the perturbation (open symbols at $S_{1}$ in Fig. 5a). Thus, in this sample of healthy elderly, longer-step responses (required after phase-delay perturbations) were more effective than shorter-step responses (required after phase-advance perturbations). This difference in the ability to accelerate and decelerate steps ${ }^{3}$ is in line with recent obstacle crossing findings (Weerdesteyn et al. 2005a, b), demonstrating an increasing proportion of longer-step over shorter-step responses with progressing age. Similarly, stroke patients show a preference for longer-step responses during obstacle crossing (Den Otter et al. 2005) and in response to rhythm perturbations in acoustically paced walking (Roerdink et al. 2009).

Interestingly, the preference for step lengthening over step shortening may provide a window into the underlying mechanisms of step adjustments and deficits therein. Varraine et al. (2000) showed that step shortening relies heavily on controlling the pendular movement of the swing leg via corticospinal pathways, whereas step lengthening is realized predominantly by an increase in propulsive forces, presumably via supraspinal structures (viz. mesencephalic and subthalamic locomotor regions). Although there are indications that these two mechanisms are non-exclusive (i.e., rapid step shortening might also involve the modulation of push-off forces (Hase and Stein 1998), whereas step lengthening was found to be partly realized by an increase in swing duration (Varraine et al. 2000)), their relative contributions clearly differ between the two gait adjustment strategies. As such, delineation of the relative contribution of these step adjustment mechanisms may be relevant from a clinical point of view. Indeed, recent results showed that stroke patients had more difficulty initiating gait adjustments with the paretic than with the non-paretic leg (Pelton et al. 2010), in particular when shorter-step responses were required (Roerdink et al. 2009). This is congruent with the fact that corticospinal pathways projecting to the paretic side are typically impaired in stroke patients.

This example shows that examining recovery characteristics following perturbations in a sequence of cues may

\footnotetext{
${ }^{3}$ Note that this difficulty to accelerate the steps was also discernable in the switching trials. More trials were excluded when a shorter-step response was required to establish synchronization with the new, phase-shifted cues (i.e., for the $-90^{\circ}$ shift; Table 1).
} 
be indicative of specific gait adjustment deficits, the identification of which may be instrumental in constructing tailored gait training programs in physical therapy or rehabilitation practice. Evidently, the ability to adjust gait in response to (changes in) the environment is important for everyday life ambulation, such as adjusting foot placement to avoid clutter or increasing speed while crossing the street when the traffic light changes to red-it represents functional and adaptive walking and relates to fall risk (e.g., Weerdesteyn et al. 2006). Eliciting gait adjustments by perturbing cueing sequences during treadmill walking may provide a fruitful means to train this ability in a safe and controlled environment in physical therapy or rehabilitation practice. In other words, this setting may be used to create a functional, task-oriented, and repetitive practice environment for training gait adjustments early in rehabilitation, allowing for the use of weight-bearing and/or handrail support (cf. Roerdink 2008). The observation that gait adjustments in healthy elderly were elicited more effectively with stepping stones than metronome beeps suggests that such a functional form of gait training would fare best using this cueing type.

An additional advantage of the use of visual stepping stones in functional gait training resides in their potential to influence gaze behavior. Often, gaze behavior is deteriorated in groups with elevated fall risks, leading to less accurate foot placement (Chapman and Hollands 2006, 2007). For example, elderly with high fall risk often adopt a potentially hazardous gaze strategy when challenged with multiple obstacles or stepping targets in the walkway, demonstrating premature gaze transfers before the ongoing step is completed (Chapman and Hollands 2007). In other words, high-risk elderly prioritize the planning of future steps over the accurate execution of ongoing steps (Chapman and Hollands 2006), which may contribute to trips and falls in cluttered environments. The use of (perturbations in the presentation of) visual stepping stones-as presented in the present study-in combination with tailored therapeutic instructions may be instrumental in training effective and safe gaze behavior during everyday ambulation, thereby putatively lowering fall risk.

Switching trials were included in the present study to directly assess the relative efficacy of the two cueing types to modify gait, although-in contrast to the perturbations-the applicability of the switching paradigm for gait rehabilitation training seems limited. The results of the switching trials were straightforward and consistent with the hypothesis: switches from metronome beeps to stepping stones were faster than vice versa (Figs. $4 c$, d, and $5 b)$. Note that the uneven distribution of excluded trials in the switching conditions (Table 1) indicated that the stepping stones were more likely to induce the intended response than the metronome beeps, a difference probably related to the salience of the distinction between cues for left and right footfalls (viz. spatial difference between the stepping stones vs. difference in pitch between the metronome beeps). This salience issue notwithstanding, a significant proportion of the excluded trials $(18 \%)$ were due to small positional changes on the treadmill that occurred after the introduction of the new, phase-shifted cues. This allowed the participant to match footfalls to both the metronome beeps and the stepping stones simultaneously. Although these positional changes were of course undesirable in our experiment, their occurrence seems to indicate a strong preference (of at least some participants) to coordinate gait with both cues at the same time. Hence, it is conceivable that the beneficial effects of either cueing type on gait (Ford et al. 2010; Hurt et al. 1998; Lewis et al. 2000; Martin 1967; McIntosh et al. 1997; Morris et al. 1994, 1996; Nieuwboer et al. 2007; Roerdink et al. 2007; Suteerawattananon et al. 2004; Thaut et al. 1997, 2007) may be amplified if these cues are presented simultaneously.

Acknowledgments The authors are grateful to Bert Coolen for developing and implementing the software for movement-dependent presentation and manipulation of metronome beeps and visual stepping stones. The contribution of Melvyn Roerdink was supported by Veni grant 451-09-024 of the Netherlands Organization for Scientific Research (NWO). Linda Bank is now at Department of Neurology, Leiden University Medical Center.

Conflict of interest The authors declare that they have no conflict of interest.

Open Access This article is distributed under the terms of the Creative Commons Attribution Noncommercial License which permits any noncommercial use, distribution, and reproduction in any medium, provided the original author(s) and source are credited.

\section{References}

Amatachaya S, Keawsutthi P, Amatachaya P, Manimmanakorn N (2009) Effects of external cues on gait performance in independent ambulatory incomplete spinal cord injury patients. Spinal Cord 47:668-673

Bertram EA, Ruina A (2001) Multiple walking speed-frequency relations are predicted by constrained optimization. J Theor Biol 209:445-453

Chapman GJ, Hollands MA (2006) Evidence for a link between changes to gaze behaviour and risk of falling in older adults during adaptive locomotion. Gait Posture 24:288-294

Chapman GJ, Hollands MA (2007) Evidence that older adult fallers prioritise the planning of future stepping actions over the accurate execution of ongoing steps during complex locomotor tasks. Gait Posture 26:59-67

Chapman GJ, Hollands MA (2010) Age-related differences in visual sampling requirements during adaptive locomotion. Exp Brain Res 201:467-478 
Chen HY, Wing AM, Pratt D (2006) The synchronisation of lower limb responses with a variable metronome: the effect of biomechanical constraints on timing. Gait Posture 23:307-314

Den Otter AR, Geurts ACH, de Haart M, Mulder T, Duysens J (2005) Step characteristics during obstacle avoidance in hemiplegic stroke. Exp Brain Res 161:180-192

Field AP (2005) Discovering statistics using SPSS, 2nd edn. Sage Publications, London, UK

Ford MP, Malone LA, Nyikos I, Yelisetty R, Bickel CS (2010) Gait training with progressive external auditory cueing in persons with Parkinson's disease. Arch Phys Med Rehabil 91:1255-1261

Hase K, Stein RB (1998) Analysis of rapid stopping during human walking. J Neurophysiol 80:255-261

Hofstad CJ, van der Linde H, Nienhuis B, Weerdesteyn V, Duysens J, Geurts AC (2006) High failure rates when avoiding obstacles during treadmill walking in patients with a transtibial amputation. Arch Phys Med Rehabil 87:1115-1122

Hollands MA, Marple-Horvat DE (2001) Coordination of eye and leg movements during visually guided stepping. J Mot Behav 33:205-216

Hollands MA, Marple-Horvat DE, Henkes S, Rowan AK (1995) Human eye movements during visually guided stepping. J Mot Behav 27:155-163

Hollands MA, Patla AE, Vickers JN (2002) "Look where you're going!": gaze behaviour associated with maintaining and changing the direction of locomotion. Exp Brain Res 143:221-230

Hurt CP, Rice RR, McIntosh GC, Thaut MH (1998) Rhythmic auditory stimulation in gait training for patients with traumatic brain injury. J Music Ther 35:228-241

Kelso JAS, Scholz JP, Schöner G (1988) Dynamics governs switching among patterns of coordination in biological movement. Phys Lett A 134:8-12

Kennedy PM, Carlsen AN, Inglis JT, Chow R, Franks IM, Chua R (2003) Relative contributions of visual and vestibular information on the trajectory of human gait. Exp Brain Res 153:113-117

Keppel G (1991) Design and analysis: a researcher's handbook, 3rd edn. Prentice-Hall, Upper Saddle River, NJ

Lewis GN, Byblow WD, Walt SE (2000) Stride length regulation in Parkinson's disease: the use of extrinsic, visual cues. Brain 123:2077-2090

Lim I, van Wegen E, de Goede C et al (2005) Effects of external rhythmical cueing on gait in patients with Parkinson's disease: a systematic review. Clin Rehabil 19:695-713

Martin JP (1967) Locomotion and the basal ganglia. In: Martin JP (ed) The basal ganglia and posture. Pitman Medical, London

McIntosh GC, Brown SH, Rice RR, Thaut MH (1997) Rhythmic auditory-motor facilitation of gait patterns in patients with Parkinson's disease. J Neurol Neurosurg Psychiatry 62:22-26

Morris ME, Iansek R, Matyas TA, Summers JJ (1994) The pathogenesis of gait hypokinesia in Parkinson's disease. Brain 117:1169-1181

Morris ME, Iansek R, Matyas TA, Summers JJ (1996) Stride length regulation in Parkinson's disease: normalization strategies and underlying mechanisms. Brain 119:551-568
Nieuwboer A, Kwakkel G, Rochester L et al (2007) Cueing training in the home improves gait-related mobility in Parkinson's disease: the RESCUE trial. J Neurol Neurosurg Psychiatry 78:134-140

Parvataneni K, Ploeg L, Olney SJ, Brouwer B (2009) Kinematic, kinetic and metabolic parameters of treadmill versus overground walking in healthy older adults. Clin Biomech 24:95-100

Patla AE (1997) Understanding the roles of vision in the control of human locomotion. Gait Posture 5:54-69

Pelton TA, Johannsen L, Chen H, Wing AM (2010) Hemiparetic stepping to the beat: asymmetric response to metronome phase shift during treadmill gait. Neurorehabil Neural Repair 24:428-434

Roerdink M (2008) Anchoring: moving from theory to therapy. Published Dissertation, (C) M. Roerdink, Amsterdam

Roerdink M, Lamoth CJC, Kwakkel G, van Wieringen PCW, Beek PJ (2007) Gait coordination after stroke: benefits of acoustically paced treadmill walking. Phys Ther 87:1009-1022

Roerdink M, Coolen H, Clairbois HE, Lamoth CJC, Beek PJ (2008) Online gait event detection using a large force platform embedded in a treadmill. J Biomech 41:2628-2632

Roerdink M, Lamoth CJC, van Kordelaar J et al (2009) Rhythm perturbations in acoustically paced treadmill walking after stroke. Neurorehabil Neural Repair 23:668-678

Rubinstein TC, Giladi N, Hausdorff JM (2002) The power of cueing to circumvent dopamine deficits: a review of physical therapy treatment of gait disturbances in Parkinson's disease. Mov Disord 17:1148-1160

Suteerawattananon M, Morris GS, Etnyre BR, Jankovic J, Protas EJ (2004) Effects of visual and auditory cues on gait in individuals with Parkinson's disease. J Neurol Sci 219:63-69

Thaut MH, McIntosh GC, Rice RR (1997) Rhythmic facilitation of gait training in hemiparetic stroke rehabilitation. J Neurol Sci 151:207-212

Thaut MH, Leins AK, Rice RR et al (2007) Rhythmic auditory stimulation improves gait more than NDT/Bobath training in near-ambulatory patients early poststroke: a single-blind, randomized trial. Neurorehabil Neural Repair 21:455-459

Varraine E, Bonnard M, Pailhous J (2000) Intentional on-line adaptation of stride length in human walking. Exp Brain Res 130:248-257

Wass E, Taylor NF, Matsas A (2005) Familiarisation to treadmill walking in unimpaired older people. Gait Posture 21:72-79

Weerdesteyn V, Nienhuis B, Duysens J (2005a) Advancing age progressively affects obstacle avoidance skills in the elderly. Hum Mov Sci 24:865-880

Weerdesteyn V, Nienhuis B, Mulder T, Duysens J (2005b) Older women strongly prefer stride lengthening to shortening in avoiding obstacles. Exp Brain Res 161:39-46

Weerdesteyn V, Rijken H, Geurts ACH, Smits-Engelsman BCM, Mulder T, Duysens J (2006) A five-week exercise program can reduce falls and improve obstacle avoidance in the elderly. Gerontology 51:131-141 